\title{
Comparing the Rates of Dopamine Hemodynamic Effect Onset after Infusion through Peripheral Veins in Three Regions
}

\author{
Deokkyu Kim ${ }^{1}$, Ji-Seon Son ${ }^{1}$, Won-Young Choi ${ }^{1}$, Young-Jin Han ${ }^{1}$, Jun-Rae Lee ${ }^{2}$, and Hyungsun Lim ${ }^{1}$ \\ ${ }^{1}$ Department of Anesthesiology and Pain Medicine, Chonbuk National University Hospital, Chonbuk National University Medical School, Jeonju; \\ 2Department of Oral and Maxillofacial Surgery, Chonbuk National University School of Dentistry, Jeonju, Korea
}

Background: Dopamine is an inotropic agent that is often selected for continuous infusion. For hemodynamic stability, the rate of infusion is controlled in the range of $5-15 \mu \mathrm{g} / \mathrm{kg} / \mathrm{min}$. This study aimed to compare the time intervals from the administration of dopamine to the onset of its hemodynamic effects when dopamine was administered through three different peripheral veins (the cephalic vein [CV], the great saphenous vein [GSV], and the external jugular vein [EJV]).

Methods: Patients in group 1, group 2, and group 3 received dopamine infusions in the CV, GSV, and EJV, respectively. A noninvasive continuous cardiac output monitor (NICCOMO ${ }^{\mathrm{m}}$, Medis, Ilmenau, Germany) was used to assess cardiac output (CO) and systemic vascular resistance (SVR). Six minutes after intubation, baseline heart rate (HR), systolic blood pressure (BP), diastolic BP, mean arterial pressure (MAP), CO, and SVR values were recorded and dopamine infusion was initiated at a dose of $10 \mu \mathrm{g} / \mathrm{kg} / \mathrm{min}$. Hemodynamic changes at $0,4,8,12$, and 15 minutes postinfusion were recorded.

Results: No statistically significant differences were observed among the three groups with respect to the rate of hemodynamic change. In all groups, systolic BP, diastolic BP, MAP, and SVR tended to increase after decreasing for the first 4 minutes; in contrast, HR and $\mathrm{CO}$ decreased until 8 minutes, after which they tended to reach a plateau.

Conclusions: For patients under general anesthesia receiving dopamine at $10 \mu \mathrm{g} / \mathrm{kg} / \mathrm{min}$, there were no clinical differences in the effect of dopamine administered through three different peripheral veins.

Key Words: administration routes; dopamine; hemodynamic status.

\section{Introduction}

Hypotension is common during induction and maintenance of general anesthesia. This condition can be caused by inhibition of central nervous system activation [1], surgical bleeding, or weakened compensation mechanisms from the effects of anesthesia. Inotropic agents can be used to treat hypotension and achieve hemodynamic stability [2]. However, if the effect of the inotropes is delayed, the risk of cardiovascular collapse may be increased. Dopamine is an inotropic agent that is often used for continuous infusion. To achieve hemodynamic stability, the infusion rate is controlled in the range of $5-15 \mu \mathrm{g} / \mathrm{kg} / \mathrm{min}[2]$.

To administrate fluids or drugs during anesthesia, intravenous routes should be secured at the patient's extremities or

\footnotetext{
Received on September 6, 2016 Revised on November 15, 2016 Accepted on November 21, 2016

Correspondence to: Ji-Seon Son, Department of Anesthesiology and Pain Medicine, Chonbuk National University Hospital, Chonbuk National University Medical School,

20 Geonij-ro, Deokjin-gu, Jeonju 54907, Korea

Tel: +82-63-250-1246, Fax: +82-63-250-1240, E-mail: sjs6803@jbnu.ac.kr
}

${ }^{\star}$ No potential conflict of interest relevant to this article was reported.

(c) This is an Open Access article distributed under the terms of the Creative Commons Attribution Non-Commercial License (http://creativecommons.org/ licenses/by-nc/4.0/) which permits unrestricted non-commercial use, distribution, and reproduction in any medium, provided the original work is properly cited. Copyright (c) 2017 The Korean Society of Critical Care Medicine 
neck. One study performed in a canine model found that circulation time after central venous injection was significantly shorter than that after peripheral venous injection during cardiac arrest [3]. The authors hypothesized that dopamine would exert its effects more rapidly in the central veins. We set out to determine whether the latency times from dopamine administration to the beginning of the hemodynamic effect differed among patients given dopamine via three different veins, the cephalic vein (CV), the great saphenous vein (GSV), and the external jugular vein (EJV). We reasoned that, if such a difference existed, this information would be an important consideration in an emergency situation.

Here we evaluated the time intervals from the administration of dopamine to the onset of the hemodynamic effect after dopamine administration via different routes.

\section{Materials and Methods}

This study was conducted at a single center (Chonbuk National University Hospital). The protocol was approved by the Institutional Review Board. Written informed consent was obtained from all patients. The minimum sample size was determined to be 56 by ANOVA. Sample size calculations were performed using SigmaPlot for Windows version 12.5 (Systat Software Inc., San Jose, CA, USA) based on the minimum detectable difference in means $(0.1)$, expected standard deviation of residuals (0.15), number of groups (3), desired power $(0.9)$, and alpha value (0.05) found in a pilot study.

This study was designed in a prospective, randomized, and double-blind fashion. Data were collected from a total of 60 adults with American Society of Anesthesiologists physical status I-II who were scheduled for elective surgery and who were aged 20-70 years. The exclusion criteria were a history of cardiovascular disease or allergy to the study drugs, use of drugs affecting vasomotor function, presence of endocrine-related cancers with vasomotor effects, and pregnancy.

No premedication was allowed before the anesthesia.
Patients were randomly assigned to one of three groups using a computerized random number generator. The $\mathrm{CV}$, GSV, and EJV groups were infused in the CV, GSV, and EJV, respectively. Standard monitoring devices for vital signs were applied and a noninvasive continuous cardiac output monitor (NICCOMO ${ }^{\mathrm{TM}}$, Medis, Ilmenau, Germany) was additionally used to measure cardiac output (CO) and systemic vascular resistance (SVR). Anesthesia was induced with propofol $2 \mathrm{mg} / \mathrm{kg}$, fentanyl $1.5 \mu \mathrm{g} / \mathrm{kg}$, and rocuronium $7 \mathrm{mg} / \mathrm{kg}$ and maintained with sevoflurane 3 vol\% $\left(\mathrm{FiO}_{2} 0.5\right.$ with $\left.\mathrm{N}_{2} \mathrm{O}\right)$. Endotracheal intubation was performed after 3 minutes of manual ventilation. At six minutes after intubation, baseline heart rate (HR), systolic blood pressure (BP), diastolic BP, mean arterial pressure (MAP), CO, and SVR values were recorded and dopamine infusion was initiated at a rate of $10 \mu \mathrm{g} / \mathrm{kg} / \mathrm{min}$. A dopamine infusion line with a stop cock was connected directly to the intravenous catheter hub in all groups. Fluid was administered at a rate of $1 \mathrm{drop} / \mathrm{second}$ and hemodynamic changes were recorded at $0,4,8,12$, and 15 minutes postinfusion. All patients were maintained in a supine position. No surgical procedures were performed during the study. All hemodynamic changes were monitored without distinguishing between the veins, and were recorded by an anesthesiologist who did not participate in the study.

Dopamine infusion was initiated when systolic BP was less than $90 \mathrm{mmHg}$ or diastolic $\mathrm{BP}$ was less than $60 \mathrm{mmHg}$, or when systolic BP dropped more than 20 $\mathrm{mmHg}$ or diastolic BP dropped more than $10 \mathrm{mmHg}$ from the pre-anesthesia values. If systolic BP was more than $180 \mathrm{mmHg}$ or diastolic BP was more than 120 $\mathrm{mmHg}$, HR was more than 120 beats per minute, or an ischemic sign was found on the electrocardiogram, dopamine infusion was maintained and the patient was managed according to proper techniques.

Inter-group comparisons were performed via two-way ANOVA for repeated measures with the Holm-Sidak post hoc test. Overall group cardiovascular responses were analyzed using one-way ANOVA for repeated measures. All analyses were performed using SigmaPlot for Win- 
dows version 12.5 (Systat Software Inc., San Jose, CA, USA). Values are expressed as mean values and standard deviations or numbers of patients. A p-value $<0.05$ was considered significant.

\section{Results}

There were 19, 19, and 22 patients in the CV, GSV, and EJV groups, respectively (Table 1). All patients met the blood pressure criteria for proceeding with the study, and dopamine infusion was initiated 6 minutes after intubation for all subjects. For 2 patients in the EJV group, the study was discontinued due to high systolic BP (more than $180 \mathrm{mmHg}$ ) or rapid HR (over 120 beats per min- ute). These patients were provided proper management and excluded from the analysis. No significant differences were observed among the three groups for any of the demographic characteristics recorded. No patient had any underlying disease, with the exception of well controlled or uncomplicated diabetes mellitus.

No significant differences were observed with respect to hemodynamic baseline values among the three groups (Table 2). Similarly, no significant differences were observed with respect to hemodynamic changes among the three groups (Figure 1). Overall, systolic BP, diastolic BP, MAP, and SVR tended to increase after decreasing for the first 4 minutes in all three groups; moreover, these values increased significantly from 12 minutes onward. In contrast, $\mathrm{HR}$ and $\mathrm{CO}$ decreased until 8 minutes postin-

Table 1. Patient demographics, underlying diseases and types of surgery

\begin{tabular}{|c|c|c|c|c|}
\hline & CV group & GSV group & EJV group & $\mathrm{p}$-value \\
\hline No. of patients (M/F) & $19(8 / 11)$ & $19(11 / 8)$ & $20(9 / 11)$ & NA \\
\hline Age (years) & $59.6 \pm 10.3$ & $59.5 \pm 8.2$ & $58.1 \pm 10.7$ & 0.916 \\
\hline Height (cm) & $161.0 \pm 5.7$ & $162.5 \pm 9.6$ & $159.2 \pm 10.6$ & 0.530 \\
\hline Weight (kg) & $65.4 \pm 9.7$ & $66.1 \pm 12.7$ & $63.3 \pm 13.0$ & 0.856 \\
\hline $\begin{array}{l}\text { Underlying diseases; well controlled \& uncomplicated } \\
\text { diabetes mellitus /NO }\end{array}$ & $2 / 17$ & $3 / 16$ & $3 / 17$ & NA \\
\hline Laparoscopic cholecystectomy & 9 & 9 & 10 & NA \\
\hline Pelviscopic myomectomy \& hysterectomy & 3 & 3 & 6 & NA \\
\hline Discectomy \& interbody fusion & 5 & 6 & 4 & NA \\
\hline Herniorrhaphy & 2 & 1 & 0 & NA \\
\hline
\end{tabular}

The values are expressed as mean \pm SD or number of patients. There were no significant differences for demographics among the three groups. There were no underlying diseases except well controlled or uncomplicated DM.

CV: cephalic vein; GSV: great saphenous vein; EJV: external jugular vein; M: male; F: female; NA: no available; NO: no underlying diseases.

Table 2. Hemodynamic baseline values

\begin{tabular}{lccc}
\hline & CV group & GSV group & EJV group \\
\hline Systolic BP $(\mathrm{mmHg})$ & $101.8 \pm 9.3$ & $99.2 \pm 15.1$ & $103.7 \pm 9.0$ \\
Diastolic BP $(\mathrm{mmHg})$ & $61.5 \pm 8.5$ & $62.0 \pm 11.3$ & $65.0 \pm 7.5$ \\
MAP (mmHg) & $77.6 \pm 7.0$ & $75.5 \pm 11.4$ & $78.9 \pm 8.7$ \\
HR (beats/min) & $74.9 \pm 11.1$ & $71.4 \pm 8.9$ & $76.7 \pm 10.2$ \\
CO (L/min) & $5.2 \pm 0.9$ & $5.0 \pm 0.9$ & $5.5 \pm 0.9$ \\
SVR (dyn·s/cm $\left.{ }^{5}\right)$ & $1,111.0 \pm 331.0$ & $1,168.9 \pm 265.8$ & $1,035.3 \pm 289.5$ \\
\hline
\end{tabular}

The values are expressed as mean \pm SD. There were no significant differences for hemodynamic baseline values among the three groups.

CV: cephalic vein; GSV: great saphenous vein; EJV: external jugular vein; BP: blood pressure; MAP: mean arterial pressure: HR: heart rate; CO: cardiac output; SVR: systemic vascular resistance. 

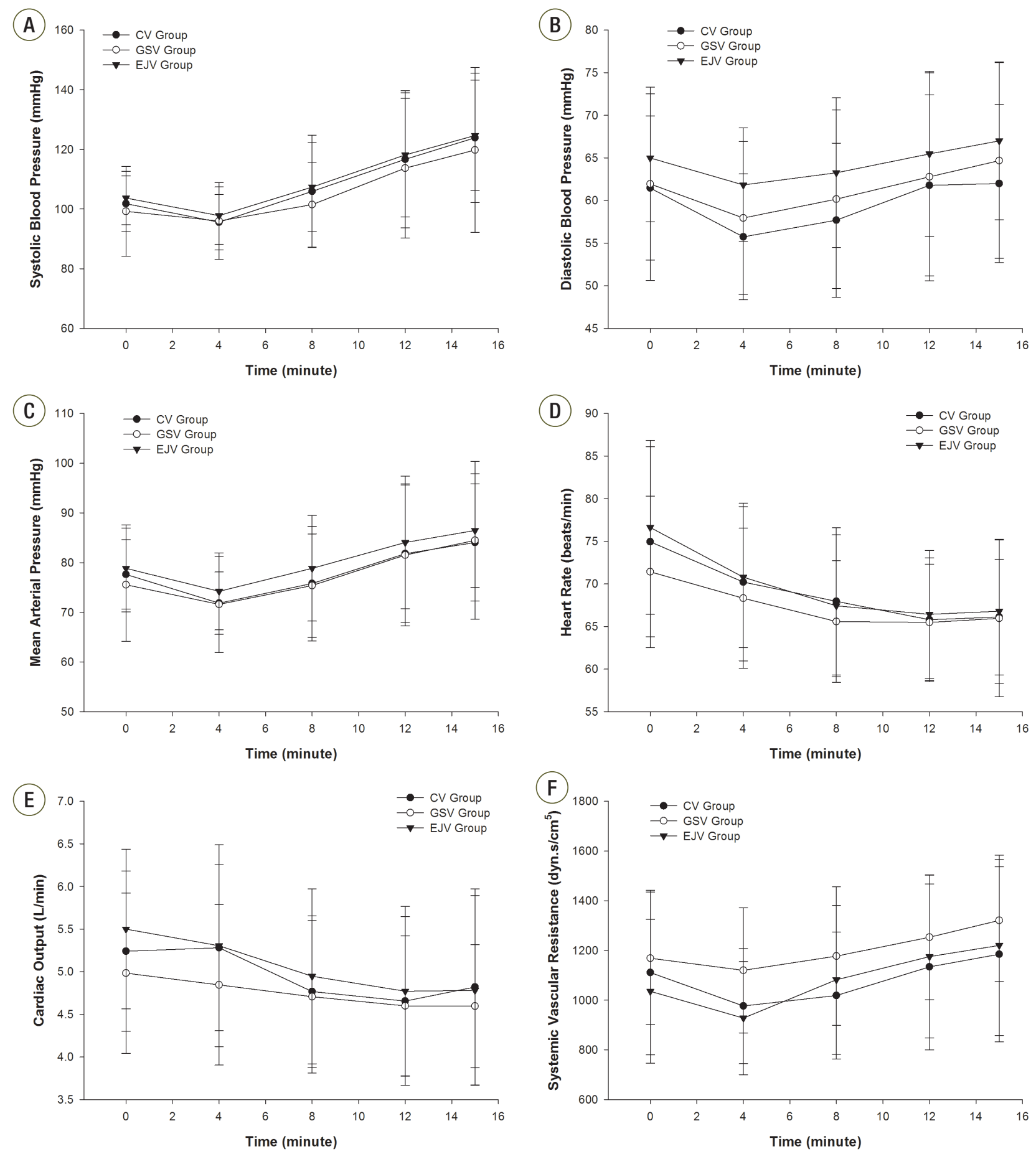

Figure 1. Hemodynamic changes over time. No significant differences were observed among the three groups. (A) Systolic blood pressure, (B) diastolic blood pressure, (C) mean arterial pressure, (D) heart rate, (E) cardiac output, (F) systemic vascular resistance. CV: cephalic vein; HR: heart rate; GSV: great saphenous vein; EJV: external jugular vein.

fusion, at which point the values tended to plateau. The hemodynamic parameters were significantly different by two-way RM ANOVA within each group. P-values re- flecting the significance of differences between observation time points are presented in Table 3. 
Table 3. Significant results by two-way RM ANOVA within each group

\begin{tabular}{|c|c|c|c|c|c|c|c|}
\hline $\begin{array}{l}\text { Hemodynamic } \\
\text { parameter }\end{array}$ & Group & $\begin{array}{l}\text { Observation time } \\
\text { (min) }\end{array}$ & $p$-value & $\begin{array}{l}\text { Hemodynamic } \\
\text { parameter }\end{array}$ & Group & $\begin{array}{l}\text { Observation time } \\
\text { (min) }\end{array}$ & $\mathrm{p}$-value \\
\hline \multirow[t]{20}{*}{ Systolic BP (mmHg) } & $\mathrm{CV}$ & Base vs. 12 min & 0.001 & HR (beats/min) & $\mathrm{CV}$ & Base vs. 4 min & 0.008 \\
\hline & & Base vs. 15 min & $<0.001$ & & & Base vs. 8 min & $<0.001$ \\
\hline & & 4 min vs. 8 min & 0.029 & & & Base vs. 12 min & $<0.001$ \\
\hline & & 4 min vs. 12 min & $<0.001$ & & & Base vs. 15 min & $<0.001$ \\
\hline & & 4 min vs. 15 min & $<0.001$ & & & 4 min vs. 12 min & 0.014 \\
\hline & & 8 min vs. 12 min & 0.028 & & & 4 min vs. 15 min & 0.022 \\
\hline & & 8 min vs. 15 min & $<0.001$ & & GSV & Base vs. 8 min & 0.009 \\
\hline & GSV & Base vs. 12 min & $<0.001$ & & & Base vs. 12 min & 0.008 \\
\hline & & Base vs. 15 min & $<0.001$ & & & Base vs. 15 min & 0.015 \\
\hline & & 4 min vs. 12 min & $<0.001$ & & EJV & Base vs. 4 min & 0.002 \\
\hline & & 4 min vs. 15 min & $<0.001$ & & & Base vs. 8 min & $<0.001$ \\
\hline & & 8 min vs. 12 min & 0.006 & & & Base vs. 12 min & $<0.001$ \\
\hline & & 8 min vs. 15 min & $<0.001$ & & & Base vs. 15 min & $<0.001$ \\
\hline & EJV & Base vs. 12 min & $<0.001$ & & & 4 min vs. 12 min & 0.033 \\
\hline & & Base vs. 15 min & $<0.001$ & $\mathrm{CO}(\mathrm{L} / \mathrm{min})$ & CV & Base vs. 12 min & 0.014 \\
\hline & & 4 min vs. 8 min & 0.005 & & & 4 min vs. 8 min & 0.040 \\
\hline & & 4 min vs. 12 min & $<0.001$ & & & 4 min vs. 12 min & 0.008 \\
\hline & & 4 min vs. 15 min & $<0.001$ & & GSV & Base vs. 8 min & 0.018 \\
\hline & & $8 \mathrm{~min}$ vs. $12 \mathrm{~min}$ & 0.001 & & & Base vs. 12 min & $<0.001$ \\
\hline & & 8 min vs. 15 min & $<0.001$ & & & Base vs. 15 min & $<0.001$ \\
\hline \multirow[t]{5}{*}{ Diastolic BP (mmHg) } & $\mathrm{CV}$ & Base vs. 4 min & 0.031 & & & 4 min vs. 12 min & 0.038 \\
\hline & & 4 min vs. 12 min & 0.022 & & & $4 \mathrm{~min}$ vs. $15 \mathrm{~min}$ & 0.040 \\
\hline & & 4 min vs. 15 min & 0.017 & & EJV & Base vs. 8 min & $<0.001$ \\
\hline & GSV & 4 min vs. 15 min & 0.002 & & & Base vs. 12 min & $<0.001$ \\
\hline & EJV & 4 min vs. 15 min & 0.007 & & & Base vs. 15 min & $<0.001$ \\
\hline \multirow[t]{14}{*}{ MAP (mmHg) } & CV & 4 min vs. 12 min & $<0.001$ & & & 4 min vs. 8 min & 0.034 \\
\hline & & 4 min vs. 15 min & $<0.001$ & & & 4 min vs. 12 min & $<0.001$ \\
\hline & & $8 \mathrm{~min}$ vs. $15 \mathrm{~min}$ & 0.008 & & & 4 min vs. 15 min & $<0.001$ \\
\hline & GSV & Baes vs. 12 min & 0.043 & SVR (dyn:s/cm5) & $\mathrm{CV}$ & 4 min vs. 15 min & 0.007 \\
\hline & & Baes vs. 15 min & 0.001 & & GSV & Base vs. 15 min & 0.002 \\
\hline & & 4 min vs. 12 min & $<0.001$ & & & 4 min vs. 12 min & 0.008 \\
\hline & & 4 min vs. $15 \mathrm{~min}$ & $<0.001$ & & & 4 min vs. $15 \mathrm{~min}$ & $<0.001$ \\
\hline & & 8 min vs. 12 min & 0.043 & & & 8 min vs. 15 min & 0.004 \\
\hline & & 8 min vs. 15 min & $<0.001$ & & EJV & Base vs. 12 min & 0.012 \\
\hline & EJV & Baes vs. 15 min & 0.002 & & & Base vs. 15 min & $<0.001$ \\
\hline & & 4 min vs. 12 min & $<0.001$ & & & 4 min vs. 8 min & 0.005 \\
\hline & & 4 min vs. 15 min & $<0.001$ & & & 4 min vs. 12 min & $<0.001$ \\
\hline & & 8 min vs. 12 min & 0.046 & & & 4 min vs. 15 min & $<0.001$ \\
\hline & & 8 min vs. 15 min & 0.001 & & & $8 \mathrm{~min}$ vs. $15 \mathrm{~min}$ & 0.011 \\
\hline
\end{tabular}

Statistically significant results of hemodynamic parameters were shown by two-way RM ANOVA within each group. BP: blood pressure; CV: cephalic vein; HR: heart rate; GSV: great saphenous vein; EJV: external jugular vein; CO: cardiac output; MAP: mean arterial pressure; SVR: systemic vascular resistance. 


\section{Discussion}

Here we investigated whether the latency times from dopamine administration to the onset of hemodynamic effects differed in patients given dopamine via three different veins (cephalic, great saphenous, and external jugular). We did not observe any differences in the latency periods from dopamine administration to hemodynamic effect onset among the different dopamine administration routes. We speculate that the hemodynamic effect of dopamine is not affected by administration route in the normal circulatory state or in the shock state. Although we did not observe any significant differences among the three groups in terms of hemodynamic changes, overall BP, systolic BP, diastolic BP, MAP, and SVR tended to increase after decreasing for 4 minutes in all groups. Moreover, these parameters all increased significantly from 12 minutes postinfusion onwards. In contrast, HR and $\mathrm{CO}$ decreased up to 8 minutes, after which the values plateaued.

Hemodynamic stability is a fundamental goal in anesthesia management. In general, hemodynamic stability promotes clinical recovery by improving $\mathrm{CO}$ and/or vascular tone when dealing with a serious threat to life. Intravenous infusion of dopamine is commonly used to maintain hemodynamic stability during anesthesia management. Dopamine, a direct precursor of norepinephrine, enables the maintenance of desirable hemodynamic properties when infused at a low to moderate rate. A previous clinical trial investigating the use of dopamine in patients with shock reported that cardiac function was improved when dopamine was used [4]. When exogenous dopamine is infused, its pharmacodynamic effects are well correlated with the rate of infusion. Specifically, dopamine has been shown to stimulate $\beta$-adrenergic receptors at intermediate doses (3-10 $\mu \mathrm{g} / \mathrm{kg} / \mathrm{min})$ and $\alpha$-adrenergic receptors at higher doses (>10 $\mu \mathrm{g} / \mathrm{kg} / \mathrm{min}$ ) [2,5]. $\beta_{1}$ receptors predominate in cardiac smooth muscle cells, whereas $\alpha_{1}$ receptors predominate in arterial smooth muscles. $\beta_{1}$ receptors act on the sinoatrial node to produce positive chronotropy and on the atrial or ventricular muscle to produce inotropy [6].
These receptors ameliorate HR (chronotropy) and cardiac contractility (inotropy) to maintain stroke volume and $\mathrm{CO}$ at a biological set point [7]. Therefore, we selected the highest possible intermediate dopamine dose $(10 \mu \mathrm{g} / \mathrm{kg} /$ min) with the goal of observing a distinct reaction of $\beta_{1}$ adrenergic receptors. However, we did not observe any increase in $\mathrm{HR}$ or $\mathrm{CO}$.

Considerable interpatient variability has been observed in dopamine pharmacokinetics, even in homogeneous populations of healthy volunteers [8,9]. Moderate doses of dopamine are thought to maximize inotropy and avoid excessive $\alpha$-adrenergic stimulation. However, definitive evidence supporting the use of dopamine in this setting is lacking. In another study, the breakpoint for a reduction in cardiac index and an increase in systemic vascular resistance index occurred at an infusion rate of approximately $15 \mathrm{mg} / \mathrm{kg} / \mathrm{min}$ [9]. Dopamine infused at a rate close to $10 \mu \mathrm{g} / \mathrm{kg} / \mathrm{min}$ was shown to exert a positive effect on $\mathrm{CO}$, whereas heart rate and arterial pressure barely changed [4]. High "vasopressor" doses of dopamine ( $>10 \mathrm{mg} / \mathrm{kg} / \mathrm{min}$ ) increase $\alpha_{1}$ receptor signaling and thus increase MAP activation by progressively increasing SVR without further increasing CO [10]. In our study, although we did not observe a clear trend of increased $\mathrm{CO}, \mathrm{BP}$ was observed to increase as SVR increased. We reason that an increase in $\mathrm{CO}$ was not observed because HR did not increase.

Two minutes after the beginning of dopamine infusion, plasma dopamine levels were significantly elevated compared with baseline, and dopamine approached a steadystate level within $30 \mathrm{~min}$ [11]. In one previous study, the plasma concentration of dopamine was shown to reach a steady state after 5 minutes of intravenous infusion [12]. We observed that systolic BP, diastolic BP, MAP, and SVR all tended to increase (after decreasing for the first 4 minutes) in all groups.

The major cause of cardiovascular response during intubation is tissue tension induced by the laryngoscopic blade in the supraglottic region [13]. The pressor response to laryngoscopy and intubation is mediated via sympathetic nerves [14]. Significant increases in mean 
arterial pressure have been noted 1 min after intubation, with mean arterial pressure returning to baseline values (i.e., preanesthesia) after about 5 minutes [15]. Dopamine infusion was started at 7 minutes after intubation in this study.

Dopamine has major side effects such as severe hypertension, ventricular arrhythmias, and cardiac ischemia. Severe hypertension and tachycardia occurred in two patients in our study. Appropriate management was provided for these patients, and the complications did not recur.

There is one particularly important limitation to our study. The effect of general anesthesia on circulation is more complex than our study setting was able to account for. In addition to their sympathovagal effects, general anesthetics (including propofol) have negative inotropic effects on the heart and direct vasodilatory effects. The hemodynamic changes caused by dopamine infusion may thus be blunted when the patient is in an anesthetized state. Some patients took nonsteroidal anti-inflammatory drugs (NSAIDs) prior to surgery. Prostacyclin $\left(\mathrm{PGI}_{2}\right)$ is a vasodilator that is synthesized in endothelial cells, primarily by a Cyclooxygenases-2-dependent pathway. Treatment with NSAIDs may inhibit production of $\mathrm{PGI}_{2}$ and reduce $\mathrm{PG}-\mathrm{mediated}$ inhibition of endothelin-1 production, resulting in increased peripheral resistance [16]. Hemodynamic changes caused by dopamine infusion in patients with NSAIDs may also be blunted. However, it is challenging to determine the precise effect of NSAIDs on these hemodynamic changes.

In summary, we did not observe any differences in the time dopamine took to affect patients under general anesthesia when dopamine was infused at a rate of $10 \mu \mathrm{g} / \mathrm{kg} / \mathrm{min}$.

\section{References}

1. Nigro Neto C, Costa E, Rossi R, Tardelli MA. Inhalation induction with sevoflurane in adult cardiac surgery patients. A case series. Heart Lung Vessel 2014; 6: 8-12.
2. Brunton L, Chabner B, Knollman B. Goodman and Gilman's The pharmacological basis of therapeutics. 12th ed. New York: McGraw-Hill Companies; 1990. pp 187-243.

3. Emerman CL, Pinchak AC, Hancock D, Hagen JF. Effect of injection site on circulation times during cardiac arrest. Crit Care Med 1988; 16: 1138-41.

4. Théroux P, Mizqala HF, Bourassa MG. Hemodynamic and therapeutic effects of intravenous dopamine. Can Med Assoc J 1977; 116: 645-8.

5. Overgaard CB, Dzavík V. Inotopes and vasopressors: review of physiology and clinical use in cardiovascular disease. Circulation 2008: 118: 1047-56.

6. Snapir A, Koskenvuo J, Toikka J, Orho-Melander M, Hinkka S, Saraste M, et al. Effects of common polymorphisms in the alpha1A-, alpha2B-, beta1- and beta2-adrenoreceptors on haemodynamic responses to adrenaline. Clin Sci (Lond) 2003; 104: 509-20.

7. Triposkiadis F, Karayannis G, Giamouzis G, Skoularigis J, Louridas G, Butler J. The sympathetic nervous system in heart failure physiology, pathophysiology, and clinical implications. J Am Coll Cardiol 2009; 54: 1747-62.

8. MacGregor DA, Smith TE, Prielipp RC, Butterworth JF, James RL, Scuderi PE. Pharmacokinetics of dopamine in healthy male subjects. Anesthesiology 2000; 92: 338-46.

9. Johnston AJ, Steiner LA, O'Connell DA, Chatfield DA, Gupta AK, Menon DK. Pharmacokinetics and pharmacodynamics of dopamine and norepinephrine in critically ill head-injured patients. Intensive Care Med 2004; 30: 45-50.

10. Martin C, Papazian L, Perrin G, Saux P, Gouin F. Norepinephrine or dopamine for the treatment of hyperdynamic septic shock? Chest 1993; 103: 1826-31.

11. Onasch A, Tanzeem A, Isqro F, Böning D, Strobel G. Effect of intravenous dopamine infusion on plasma concentrations of dopamine and dopamine sulfate in men, during and up to $18 \mathrm{~h}$ after infusion. Eur J Clin Pharmacol 2000; 55: 755-9.

12. Järnberg PO, Bengtsson J, Ekstrand J, Hamberger B. 
Dopamine infusion in man. Plasma catecholamine levels and pharmacokinetics. Acta Anaesthesiol Scand 1981; 25: 328-31.

13. Barak M, Ziser A, Greenberg A, Lischinsky S, Rosenberg B. Hemodynamic and catecholamine response to tracheal intubation: direct laryngoscopy compared with fiberoptic intubation. Cardiovascular and catecholamine responses to laryngoscopy with or without tracheal intubation. J Clin Anesth 2003; 15: $132-6$.

14. McCoy EP, Mirakhur RK, McCloskey BV. A com- parison of the stress response to Laryngoscopy. The Macintosh versus the McCoy blade. Anaesthesia 1995; 50: 943-6.

15. Russell WJ, Morris RG, Frewin DB, Drew SE. Changes in plasma catecholamine concentrations during endotracheal intubation. Br J Anaesth 1981; 53: 837-9.

16. Johnson AG. NSAIDs and blood pressure. Clinical importance for older patients. Drugs Aging 1998; 12: 17-27. 